\title{
AzTEC 1.1 mm OBSERVATIONS OF THE MBM12 MOLECULAR CLOUD
}

\author{
M. J. Kim ${ }^{1}$, S. Kim ${ }^{1}$, S. Youn ${ }^{1}$, M. S. Yun ${ }^{2}$, G. W. Wilson ${ }^{2}$, I. AretXaga ${ }^{3}$, J. P. Williams ${ }^{4}$, D. H. Hughes ${ }^{3}$, A. Humphrey ${ }^{3}$, \\ J. E. Austermann ${ }^{5}$, T. A. Perera ${ }^{6}$, P. D. Mauskopf ${ }^{7}$, L. Magnani ${ }^{8}$, And Y.-W. Kang ${ }^{1}$ \\ ${ }^{1}$ Department of Astronomy \& Space Science, Sejong University, KwangJin-gu, KunJa-dong 98, Seoul 143-747, Republic of Korea; sek@ sejong.ac.kr \\ ${ }^{2}$ Department of Astronomy, University of Massachusetts, 710 North Pleasant St., Amherst, MA 01003, USA \\ ${ }^{3}$ Instituto Nacional de Astrofísca, Óptica y Electrónica, Tonantzintla, Puebla, Mexico \\ ${ }^{4}$ Institute for Astronomy, University of Hawaii, 2680 Woodlawn Drive, Honolulu, HI 96822, USA \\ ${ }^{5}$ Department of Astrophysical and Planetary Sciences, University of Colorado, Boulder, Colorado 80309, USA \\ ${ }^{6}$ Department of Physics, Illinois Wesleyan University, Bloomington, IL 61701, USA \\ ${ }^{7}$ School of Physics and Astronomy, Cardiff University, Cardiff CF24 3AA, UK \\ ${ }^{8}$ Department of Physics and Astronomy, The University of Georgia, Athens, GA 30602-2451, USA \\ Received 2011 August 26; accepted 2011 November 2; published 2012 January 19
}

\begin{abstract}
We present $1.1 \mathrm{~mm}$ observations of the dust continuum emission from the MBM12 high-latitude molecular cloud observed with the Astronomical Thermal Emission Camera (AzTEC) mounted on the James Clerk Maxwell Telescope on Mauna Kea, Hawaii. We surveyed $6.34 \mathrm{deg}^{2}$ centered on MBM12, making this the largest area that has ever been surveyed in this region with submillimeter and millimeter telescopes. Eight secure individual sources were detected with a signal-to-noise ratio of over 4.4. These eight AzTEC sources can be considered to be real astronomical objects compared to the other candidates based on calculations of the false detection rate. The distribution of the detected $1.1 \mathrm{~mm}$ sources or compact $1.1 \mathrm{~mm}$ peaks is spatially anti-correlated with that of the $100 \mu \mathrm{m}$ emission and the ${ }^{12} \mathrm{CO}$ emission. We detected the $1.1 \mathrm{~mm}$ dust continuum emitting sources associated with two classical T Tauri stars, $\mathrm{LkH} \alpha 262$ and $\mathrm{LkH} \alpha 264$. Observations of spectral energy distributions (SEDs) indicate that $\mathrm{LkH} \alpha 262$ is likely to be Class II (pre-main-sequence star), but there are also indications that it could be a late Class I (protostar). A flared disk and a bipolar cavity in the models of Class I sources lead to more complicated SEDs. From the present AzTEC observations of the MBM12 region, it appears that other sources detected with AzTEC are likely to be extragalactic and located behind MBM12. Some of these have radio counterparts and their star formation rates are derived from a fit of the SEDs to the photometric evolution of galaxies in which the effects of a dusty interstellar medium have been included.
\end{abstract}

Key words: galaxies: high-redshift - ISM: molecules - stars: pre-main sequence - stars: protostars - submillimeter: galaxies - submillimeter: stars

Online-only material: color figures

\section{INTRODUCTION}

The MBM12 molecular cloud was cataloged as L1453L1454, L1457, and L1458 in Lynds' Dark Cloud Catalog (Lynds 1962) and is one of the nearby high-latitude molecular clouds (Magnani et al. 1985). Until recently, MBM12 was considered to be the nearest star-forming region; however, its distance has been revised to $275 \mathrm{pc}$ by Luhman (2001) and at $360 \pm 30 \mathrm{pc}$ from the photometric and spectroscopic results of the Sloan Digital Sky Survey (SDSS) and the Two Micron All Sky Survey (2MASS; Andersson et al. 2002). Thus, although it is still nearby, it is farther out than the Taurus dark clouds and Ophiuchus star-forming regions.

A few young stars were reported in MBM12 (Hearty et al. 2000a; Hogerheijde et al. 2003) and their presence demonstrated that star formation had occurred recently in at least this particular high-latitude molecular cloud (Broeg et al. 2006). The highlatitude molecular clouds are known to be translucent, and the low-density environments found in high-latitude clouds create a challenge for star formation by gravitational collapse. The ability of at least a few high-latitude clouds to form cold molecular cores and young stars may be due to a combination of conditions, including variations in the interstellar radiation field, changes in dust-grain size and chemistry, and turbulence in the interstellar medium (ISM).
Regardless of the formation mechanisms, the study of high-latitude star formation environments continues to intrigue researchers, because it is difficult to see how star formation in the low-density environments found in typical high-latitude molecular clouds can proceed via direct gravitational collapse. High-latitude pre-main-sequence (PMS) stars found in isolation could be formed by ejection from their parent cloud or dissipation of the natal cloud. Dissipation timescales for these unbound clouds appear to be a few Myr. It is possible that outflows from low-mass stars that form in low-density environments can drive the dissipation of the parent cloud (Sterzik \& Durisen 1995; Feigelson 1996).

In the present paper, we describe the results of our observations of the dust continuum emission from MBM12 at a wavelength of $1.1 \mathrm{~mm}$ observed with the AzTEC (Wilson et al. 2008) on the James Clerk Maxwell Telescope (JCMT). Dust continuum emission at (sub)millimeter wavelengths can identify the star-forming sites in the molecular clouds and determine the evolutionary stages of young stellar objects. In Section 2, we describe the observations and data reduction procedure. In Section 3, we present information on the sources detected at $1.1 \mathrm{~mm}$ and investigate their spectral energy distributions (SEDs). We also describe the assembly of the SEDs and the procedure used to fit the models, and how models were fitted to the data to determine the physical 


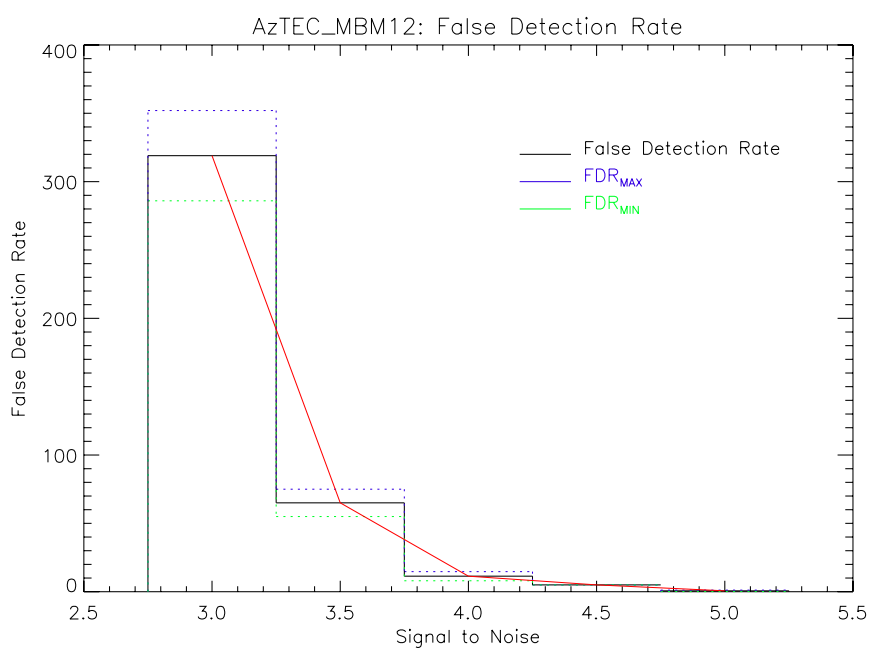

Figure 1. FDR at each threshold of $3 \sigma-5 \sigma$, each step corresponds to $0.5 \sigma$. (A color version of this figure is available in the online journal.)

parameters of the detected sources from present observations. In Section 4, we summarize the results of our observations and analysis.

\section{OBSERVATIONS AND DATA REDUCTION}

Observations of MBM12 were performed in raster scan mode between 2005 November and 2006 January using AzTEC mounted on the JCMT. The beam size was 18 arcsec, and the scan velocity was $120 \operatorname{arcsec~s}^{-1}$. AzTEC is a new bolometric array instrument and was developed for the Large Millimeter Telescope at the University of Massachusetts at Amherst (Wilson et al. 2008). This array camera consists of $144 \mathrm{Si}_{3} \mathrm{~N}_{4}$ micromesh bolometers and operates at $1.1 \mathrm{~mm}$ and $2.1 \mathrm{~mm}$. In 2005, this bolometer array was tested by invitation at the JCMT on Mauna Kea in Hawaii. It is an appropriate instrument for surveys of Submillimeter Galaxies (SMGs) at low and high redshifts and searches for protostellar dusty cores in nearby molecular clouds. We surveyed about $6.34 \mathrm{deg}^{2}$ of the sky centered on R.A. $=02^{\mathrm{h}} 56^{\mathrm{m}} \cdot 0$, decl. $=19^{\mathrm{d}} 30^{\mathrm{m}} \cdot 0$ in $\mathrm{J} 2000$. This is the largest area centered on MBM12 that has ever been surveyed using continuum instruments in the submillimeter and millimeter parts of the spectrum. The area was covered with a total of 36 scan maps. Twenty-seven scans were retrieved and combined, covering a total observed area of $655.35 \mathrm{arcmin}^{2}$. The average rms was $9.695 \mathrm{mJy}$ beam $^{-1}$. AzTEC suffered a pointing error of about $2^{\prime \prime}$. Beam maps were made from observations of Uranus with a mean flux density of $42.2 \mathrm{Jy}$ at $1.1 \mathrm{~mm}$ during the JCMT observing run. A pointing model was generated using CRL618, a post-AGB (asymptotic giant branch) star with a mean flux density of $2.7 \mathrm{Jy}$ at $1.1 \mathrm{~mm}$. The intensity calibration errors were estimated to be $\pm 6 \%-13 \%$ (Wilson et al. 2008). The raw bolometer array observations were calibrated and edited in the standard AzTEC IDL software (Scott et al. 2008; Austermann et al. 2010). The AzTEC instrument team has constructed data-reduction codes in the IDL language. Using the AzTEC software, we cleaned and co-added the array observations. To remove atmospheric signals, we adopted the principal component analysis (PCA) technique (Heyer \& Schloerb 1997; Francis \& Wills 1999), a tool for simplifying several parameters to clarify their relationships. Given a sample of $n$ objects with $p$ parameters, $x_{j}(j=1, \ldots, p)$, the orthogonal
Table 1

The Calculated FDR of the AzTEC Sources in the MBM12 Regions

\begin{tabular}{lccc}
\hline \hline Area & $\begin{array}{c}\text { Signal } \\
(>5 \sigma)\end{array}$ & $\begin{array}{c}\text { Average Noise rms } \\
\left(\text { Jy beam }^{-1}\right)\end{array}$ & FDR \\
\hline A1 & 1 & 0.009 & $0.18 \pm 0.10$ \\
A2 & 2 & 0.007 & $0.19 \pm 0.81$ \\
A3 & 0 & 0.007 & 0 \\
A4 & 2 & 0.011 & $0.36 \pm 0.09$ \\
A9 & 1 & 0.013 & $0.64 \pm 0.54$ \\
\hline
\end{tabular}

variables, $\xi_{i}(i=1, \ldots, p)$, can be described as

$$
\xi_{i}=a_{i 1} x_{1}+\cdots+a_{i j} x_{j}+\cdots+a_{i p} x_{p} .
$$

The $\xi_{i}$ represent the principal component, and the correlated and observed variables flag important principal components (Brunt \& Heyer 2002). Hence, atmospheric signals with large spatial fluctuation show dominant principal components, whereas point signals such as distant galaxies do not. We adapted the PCA cleaning routine by constructing a covariance matrix from the $N_{\text {bolo }} \times N_{\text {time }}$ time-stream data (Scott et al. 2008; Austermann et al. 2010). To provide an eigenvalue decomposition on the matrix, we neglected the eigenfunctions corresponding to eigenvalues larger than $2.5 \sigma$ as atmospheric effects (Scott et al. 2008; Austermann et al. 2010). To maximize the signal-to-noise ratio $(\mathrm{S} / \mathrm{N})$, the four cleaned raw data sets were co-added with the Wiener filter, $g(q)$, described as

$$
g(q)=\frac{s^{*}(q) / J(q)}{\int|s(q)|^{2} / J(q) d^{2} q},
$$

where $s(q)$ is the Fourier transform of the beam shape from map space to spatial frequency space, $q$, and $J(q)$ implies the average power spectral densities (PSDs). Through filtering with the Wiener filter (Scott et al. 2008; Austermann et al. 2010) in Fourier space, the noise at low and high frequencies was effectively attenuated (Laurent et al. 2005). Using the Wiener filter and removing the PCA components reduces the rms noise per pixel, but the resolution of the optimally filtered map appeared to be the same. The peak brightness of point sources and compact peaks would be also preserved. However, after optimal filtering extended sources in the map will be trimmed to remove areas of low coverage (Enoch et al. 2007). The final image of MBM12 contains eight sources detected at a $\mathrm{S} / \mathrm{N}$ of greater than $4.0 \sigma$ (Figure 1). The average rms noise over the entire image is $2.71 \mathrm{mJy}^{\text {beam }}{ }^{-1}$. The catalog of the AzTEC mm source candidates is listed in Table 1 . The flux values of the source candidates range from $10 \pm 2.7 \mathrm{mJy}$ to $12 \pm 2.7 \mathrm{mJy}$.

We generated noise maps by randomly multiplying each scan in the cleaned time stream data by \pm 1 and co-adding (Perera et al. 2008; Scott et al. 2008). The sources were removed, and the random noises were accumulated in the co-added noise map (Austermann et al. 2010). We constructed 100 noise maps by iteration. We determined the false detection rate (FDR) by analyzing the noise properties of these maps by counting signals detected with a flux over a threshold in each noise map and then estimating the average detections (Perera et al. 2008). The FDR was defined as the source detections divided by the average false detections. The FDR at each threshold is shown in Figure 1. At over $3.0 \sigma, 312$ sources were detected, and the FDR was $319 \pm$ 66 . However, at over $4.4 \sigma$, eight sources were detected, and the FDR value was smaller than 1. Therefore, these eight AzTEC sources are more likely to be real astronomical objects than 
Table 2

Observed AzTEC Sources in the MBM12 Region

\begin{tabular}{|c|c|c|c|c|c|}
\hline Source & & R.A. (J2000) & Decl. (J2000) & $\begin{array}{c}\text { Flux } \\
\left(\mathrm{mJy}_{\text {beam }}^{-1}\right)\end{array}$ & $\mathrm{S} / \mathrm{N}$ \\
\hline $\mathrm{A} 1_{1}$ & $\mathrm{~J} 0225+1904$ & $02: 55: 17.3$ & $19: 04: 48$ & $45.6 \pm 3.6$ & 5.2 \\
\hline $\mathrm{A} 2{ }_{1}$ & $\mathrm{~J} 0256+2005(\mathrm{LkH} \alpha 262)$ & $02: 56: 37.5$ & $20: 05: 36$ & $87.8 \pm 7.0$ & 12.6 \\
\hline $\mathrm{A} 22_{2}$ & J0256+2003 (LkH $\alpha 264)$ & $02: 56: 07.7$ & $20: 03: 24$ & $44.9 \pm 3.6$ & 6.3 \\
\hline $\mathrm{A} 3_{1}$ & J0258+2030 & 02:58:07.3 & 20:30:00 & $29.6 \pm 2.4$ & 4.4 \\
\hline $\mathrm{A} 4_{1}$ & $\mathrm{~J} 0259+1925$ & $02: 59: 29.5$ & $19: 25: 42$ & $234.3 \pm 18.7$ & 22.7 \\
\hline $\mathrm{A} 4{ }_{2}$ & $\mathrm{~J} 0258+1953$ & $02: 58: 41.3$ & $19: 53: 18$ & $50.7 \pm 4.0$ & 5.0 \\
\hline $\mathrm{A} 99_{1}$ & $\mathrm{~J} 0253+1805$ & $02: 53: 34.8$ & $18: 05: 42$ & $298.5 \pm 23.9$ & 11.4 \\
\hline $\mathrm{A} 99_{2}$ & $\mathrm{~J} 0257+1847$ & $02: 57: 45.6$ & $18: 47: 06$ & $53.6 \pm 4.2$ & 4.4 \\
\hline
\end{tabular}

Notes. Units of R.A. are in hours, minutes, and seconds. Units of decl. are in degrees, arcminutes, and arcseconds. The positional uncertainties of sources are likely to be of order of $1^{\prime \prime}$ in R.A. and decl..

the others. The results from the calculations of the FDR are summarized in Table 1.

\section{RESULTS AND DISCUSSION}

\subsection{Distribution of the AzTEC $1.1 \mathrm{~mm}$ Dust Continuum Sources}

Figure 2 presents the $1.1 \mathrm{~mm}$ dust continuum emitting AzTEC sources from the present AzTEC MBM12 survey. The ${ }^{12} \mathrm{CO}(1-0)$ contours from the AT\&T Bell Lab, $7 \mathrm{~m}$ radio telescope survey of MBM12 (Pound et al. 1990) are shown overlaid on the IRAS $100 \mu \mathrm{m}$ emission in Figure 3. It is clear from the figure that the ${ }^{12} \mathrm{CO}$ contours are spatially correlated with the $100 \mu \mathrm{m}$ emission. The AzTEC $1.1 \mathrm{~mm}$ dust continuum sources appear to be spatially anti-correlated with the most intense ${ }^{12} \mathrm{CO}$ emitting region and, thus, with the more intense $100 \mu \mathrm{m}$ IRAS regions in the cloud. The lack of AzTEC mm sources and compact peaks in the bright $100 \mu \mathrm{m}$ emitting cores suggests that our AzTEC sources detected with the $1.1 \mathrm{~mm}$ bolomoter camera trace a colder dust component in the region than the extragalactic sources. Figure 4 shows the $1.1 \mathrm{~mm}$ dust continuum AzTEC sources overlaid on the images of the $100 \mu \mathrm{m}$ emission. The anti-correlation between the AzTEC dust continuum sources and the peaks of $100 \mu \mathrm{m}$ IRAS and ${ }^{12} \mathrm{CO}(1-0)$ emission is clear from the figure.

\subsection{Properties of the AzTEC Sources in the MBM12}

We present eight AzTEC millimeter sources in Figure 2. These sources are overlaid on the IRAS $100 \mu \mathrm{m}$ image in Figure 3. The catalog of detected sources is presented in Table 2. The sources in the catalog were detected with a $\mathrm{S} / \mathrm{N}$ of over $4.4 \sigma$. Each point source is indicated on the co-added map of the MBM 12 region. The size of the circle in the box is 18 arcsec, which is the beam size of the AzTEC observations for the present survey. To identify the nature of these sources and their properties, we examined their SEDs. The intrinsic flux densities of the detected AzTEC sources were used for the analysis of their SEDs (Perera et al. 2008). Among the eight AzTEC sources, two sources (A2 $1, A 22)$ in Table 1 correspond to known T Tauri stars, $\mathrm{LkH} \alpha 262$ and $\mathrm{LkH} \alpha$ 264, with strong $\mathrm{H} \alpha$ emission and Li I absorption (Hearty et al. 2000a; Luhman 2001). Studies of seven $\mathrm{T}$ Tauri stars in this region were conducted previously (Table 3).
Table 3

Previously Observed T Tauri Stars in the MBM12

\begin{tabular}{lccc}
\hline \hline Star & R.A. (J2000) & Decl. (J2000) & Ref. \\
\hline RXJ0255.4+2005 & $02: 55: 25.7$ & $20: 04: 53$ & 6 \\
LkH $\alpha$ 262 & $02: 56: 07.9$ & $20: 03: 25$ & 1,2 \\
LkH $\alpha$ 263 & $02: 56: 08.4$ & $20: 03: 39$ & 1,2 \\
LkH $\alpha$ 264 & $02: 56: 37.5$ & $20: 05: 38$ & $1,2,3,4$ \\
E02553+2018 & $02: 58: 11.2$ & $20: 30: 04$ & 2,5 \\
RXJ0258.3+1947 & $02: 58: 15.9$ & $19: 47: 17$ & 6 \\
RXJ0256.3+2005 & $02: 56: 17.9$ & $20: 06: 10$ & 6 \\
\hline
\end{tabular}

Notes. Units of R.A. are in hours, minutes, and seconds. Units of decl. are in degrees, arcminutes, and arcseconds.

References. (1) Herbig \& Bell 1988; (2) Fernández et al. 1995; (3) Magnani et al. 1995; (4) Gameiro et al. 1993; (5) Caillault et al. 1995; (6) Hearty et al. 2000a.

\subsubsection{AzTEC Sources with Lower FDR}

$J 0256+2005\left(A 2_{1}\right)$ and $J 0256+2003\left(A 2_{2}\right)$ : we detected $1.1 \mathrm{~mm}$ dust continuum emitting sources associated with $\mathrm{LkH} \alpha$ 262 (J0256+2005) and LkH $\alpha 264$ (J0256+2003). Previous studies by Hogerheijde et al. (2003) reported observing continuum emission from the disks around the classical T Tauri stars $\mathrm{LkH} \alpha$ 262, $\mathrm{LkH} \alpha 263$, and $\mathrm{LkH} \alpha 264$ at $450 \mu \mathrm{m}$ and $850 \mu \mathrm{m}$ with Submillimeter Common User Bolometer Array (SCUBA). These two stars, $\mathrm{LkH} \alpha 262$ and $\mathrm{LkH} \alpha 264$ (Itoh et al. 2003), emitting submillimeter and millimeter radiation, were reported as Class II (Hearty et al. 2000a; Luhman 2001; Hogerheijde et al. 2003). The SEDs in Figure 5 indicate that $\mathrm{LkH} \alpha 262$ is likely to be Class II, but there are also indications that this source might be a late Class I (André et al. 1993; Whitney et al. 2003) instead. The flux flattens toward the longer wavelengths, a phenomenon that can be seen in the late Class I. To provide a reasonable fit to the flux densities between 3.6 and $8.0 \mu \mathrm{m}$ for the SED of $\mathrm{LkH} \alpha$ 262, we used a one-dimensional radiative transfer model of DUSTY (Ivezic et al. 1999). The physical properties of the central source and the dusty envelope were derived from the modeling. The flux densities from the central source were absorbed by the dusty envelope and re-emitted to longer wavelengths. A dusty disk was considered in order to fit $24 \mu \mathrm{m}$ and $70 \mu \mathrm{m}$ wavelengths, since it was difficult to fit the flux densities using the stellar component. A power-law density distribution for the envelope was also tested for emission from the disk model. The estimated mass of the envelope was consistent with the mass of the envelope determined from the $1.1 \mathrm{~mm}$ continuum by fixing most of the free parameters. The 

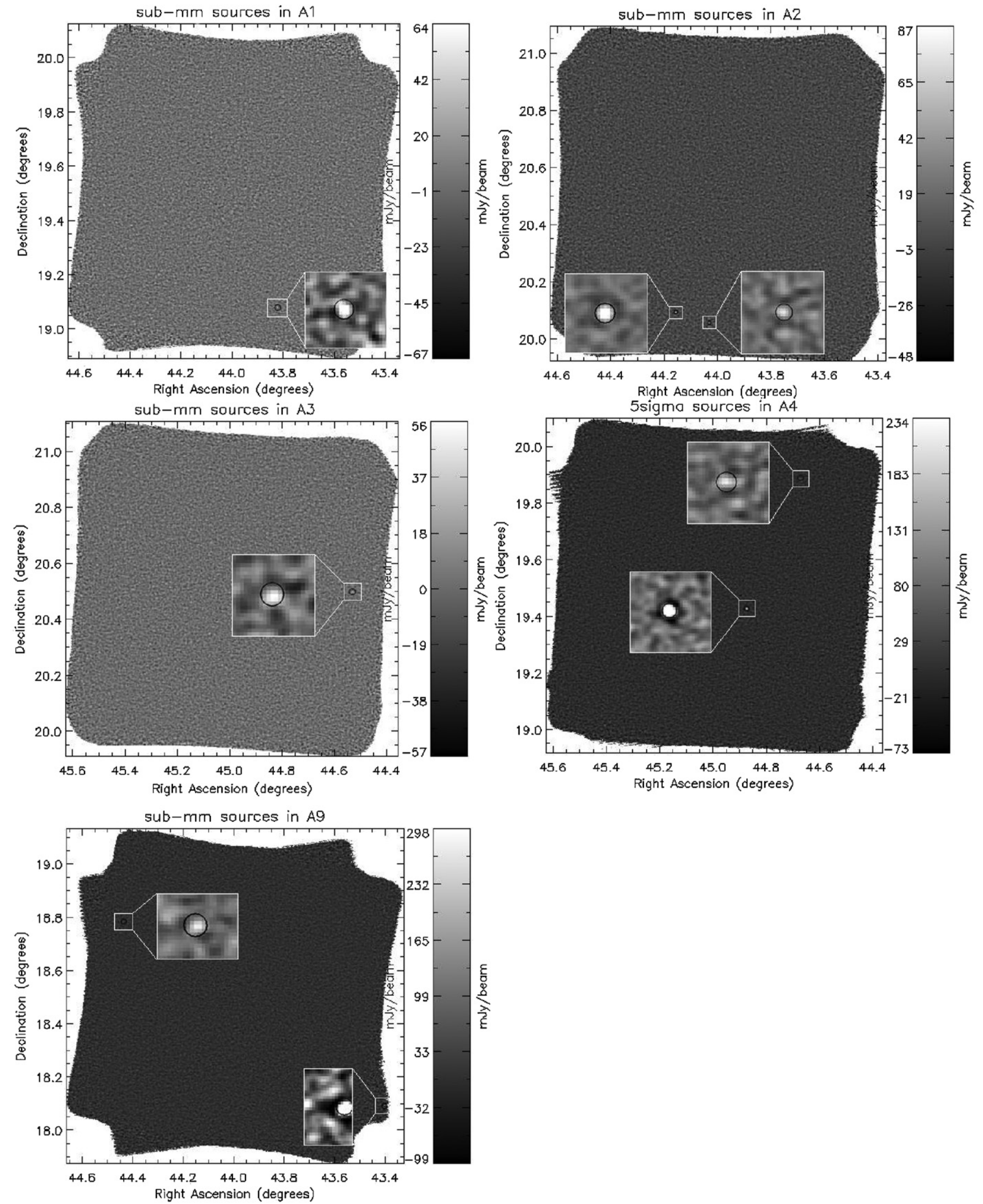

Figure 2. Eight AzTEC sources listed in Table 2 detected in the five regions covering the MBM12 high-latitude cloud. 


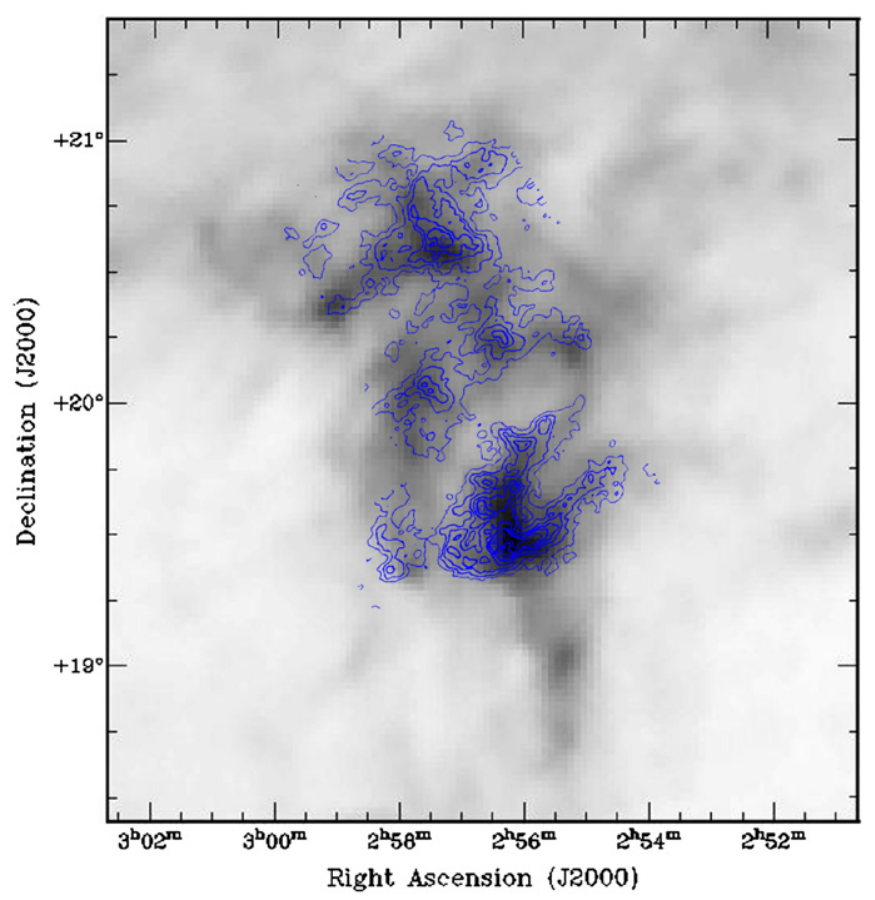

Figure 3. ${ }^{12} \mathrm{CO}$ contours from Pound et al. (1990) are overlaid on the IRAS $100 \mu \mathrm{m}$ image of the MBM12 cloud.

mass of the envelope was determined from the flux density $S$ at $1.1 \mathrm{~mm}$, the Planck function at temperature $T$, and the dust opacity $\kappa$. The dust opacities used in this analysis were from OH5 dust in Ossenkopf \& Henning (1994). The estimated mass was about $0.04 M_{\odot}$ in the $15-20 \mathrm{~K}$ temperature range. Our detections might suggest that at least the disks of the classical $\mathrm{T}$ Tauri stars in MBM12 (1-5 Myr) have a mass range similar to the mass range of the disks of the younger $(\sim 1$ Myr $)$ Taurus and $\rho$ Ophiuchus regions $\left(0.001-0.3 M_{\odot}\right.$; Andrews \& Williams 2007; Williams \& Cieza 2011). Luhman (2001) argued that the near-IR $K$ - and $L$-band excesses indicated that significant disk dispersal had occurred in MBM12.

\subsubsection{AzTEC Sources with Higher FDR}

RXJ0255.4+2005: this object was classified as a weak-line T Tauri star (WTTS). Its spectral type was labeled as K6 by Hearty et al. (2000b). $T_{\text {eff }}$ was reported as $4205 \mathrm{~K}$ by Luhman (1999) and $4275 \mathrm{~K}$ by Hearty et al. (2000b). Hogerheijde et al. (2002) calculated the disk mass and diameter using the Owens Valley Radio Observatory (OVRO) to observe the $\lambda=3 \mathrm{~mm}$ continuum. The results were reported as $0.04 M_{\odot}$ and $52 \mathrm{AU}$, respectively. These values were based on a distance of $275 \mathrm{pc}$ and a gas-to-dust ratio of 100 . The disk mass could be $0.023 M_{\odot}$ at the distance of $360 \mathrm{pc}$. There were also studies by Hearty et al. (2000b) and Herbst et al. (2004) for the line widths of $\mathrm{H} \alpha$ from the source. The emission line widths were $-1.26 \AA$ (Hearty et al. 2000b) and $-1.1 \AA$ (Herbst et al. 2004). The flux of this object varies, and the period was reported as 6.22 days by Herbst et al. (2004) and $3.36 \pm 0.17$ days by Broeg et al. (2006).

$E 02553+2018$ : this object appears on the border between a classical and a WTTS. The emission line width of $\mathrm{H} \alpha$ is about $-1.6 \AA$ (Hearty et al. 2000b) and $-2.5 \AA$ (Herbst et al. 2004). Its spectral type is K3 (Fleming et al. 1989) or K4 (Hearty et al. 2000b). The effective temperature, $T_{\text {eff }}$, of this object is $4660 \mathrm{~K}$ as calculated by Luhman (1999) and $4593 \mathrm{~K}$ by Hearty et al. (2000b). Hogerheijde et al. (2002) obtained a disk mass

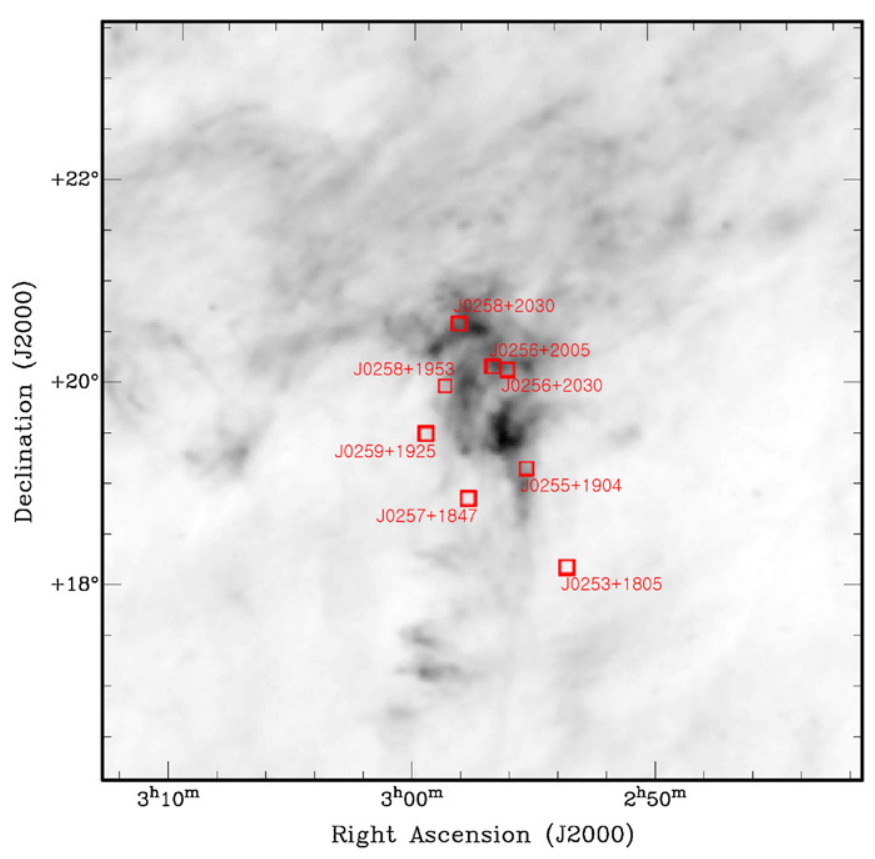

Figure 4. Eight AzTEC sources listed in Table 2 overlaid on the IRAS $100 \mu \mathrm{m}$ image.

of $0.07 M_{\odot}$ and a diameter of $66 \mathrm{AU}$. The flux was measured as $11 \mathrm{mJy}$ with OVRO. The disk mass could be $0.04 M_{\odot}$ at the distance of $360 \mathrm{pc}$. This object has an additional component of $V_{\mathrm{LSR}}=-1.5 \mathrm{~km} \mathrm{~s}^{-1}$.

RXJ0258.3+1947: this object was classified as a classical T Tauri star (CTTS). Its spectral type was determined as M5 (Hearty et al. 2000b) or M4.5 (Luhman 2001). The effective temperature, $T_{\text {eff }}$, was calculated as $3198 \mathrm{~K}$ by Luhman (1999) and $3169 \mathrm{~K}$ by Hearty et al. (2000b). The flux at $3 \mathrm{~mm}$ was $5 \mathrm{mJy}$ and the disk mass was $0.05 M_{\odot}$. The disk mass could be $0.03 M_{\odot}$ at the distance of $360 \mathrm{pc}$. Its diameter was $58 \mathrm{AU}$ from OVRO observations, and JCMT observations yielded a mass of $8 \times 10^{-4} M_{\odot}$ (Hogerheijde et al. 2002). Its flux varies, and the period was reported as 1.205 days (Herbst et al. 2004). This was confirmed with reports of an $\mathrm{H} \alpha$ emission line width of $-24.5 \AA$ from Hearty et al. (2000b) and $-25 \sim-34 \AA$ from Herbst et al. (2004).

RXJ0256.3+2005: this object was classified as a WTTS and is the latest spectral type in the field of MBM12. Its spectral type was designated as M5.75 (Luhman 2001) and M6 (Herbst et al. 2004). Luhman (1999) calculated that the effective temperature, $T_{\text {eff }}$, was $3024 \mathrm{~K}$. The mass was between 0.15 and $0.1 M_{\odot}$ (Meeus et al. 2009). Its $\mathrm{H} \alpha$ emission line width was $-13.5 \AA$ (Herbst et al. 2004).

\subsection{Properties of the AzTEC Sources behind the MBM12}

From the present AzTEC observations of the MBM12 region, we find that other detected sources are likely to be located behind MBM12. Four AzTEC objects, defined as J0258+2030 $\left(\mathrm{A} 3_{1}\right), \mathrm{J} 0259+1925\left(\mathrm{~A} 4_{1}\right), \mathrm{J} 0253+1805\left(\mathrm{~A} 9_{1}\right)$, and J0257+1847 $\left(\mathrm{A} 9{ }_{2}\right)$, were previously studied in the $1.4 \mathrm{GHz}$ NRAO VLA survey for the sky of $\delta \geqslant-40^{\circ}$ in $\mathrm{J} 2000$ (Condon et al. 1998). $\mathrm{J} 0259+1925\left(\mathrm{~A}_{1}\right)$ is known as quasi-stellar object (QSO) at a redshift of $z=0.54$. The flux density of this object at 1.4 GHz was $169.9 \mathrm{mJy}$. Three AzTEC sources, J0258+2030 $\left(\mathrm{A} 3_{1}\right), \mathrm{J} 0253+1805\left(\mathrm{~A} 9_{1}\right)$, and $\mathrm{J} 0257+1847\left(\mathrm{~A} 9_{2}\right)$, have radio counterparts observed by Condon et al. (1998) with the VLA at 

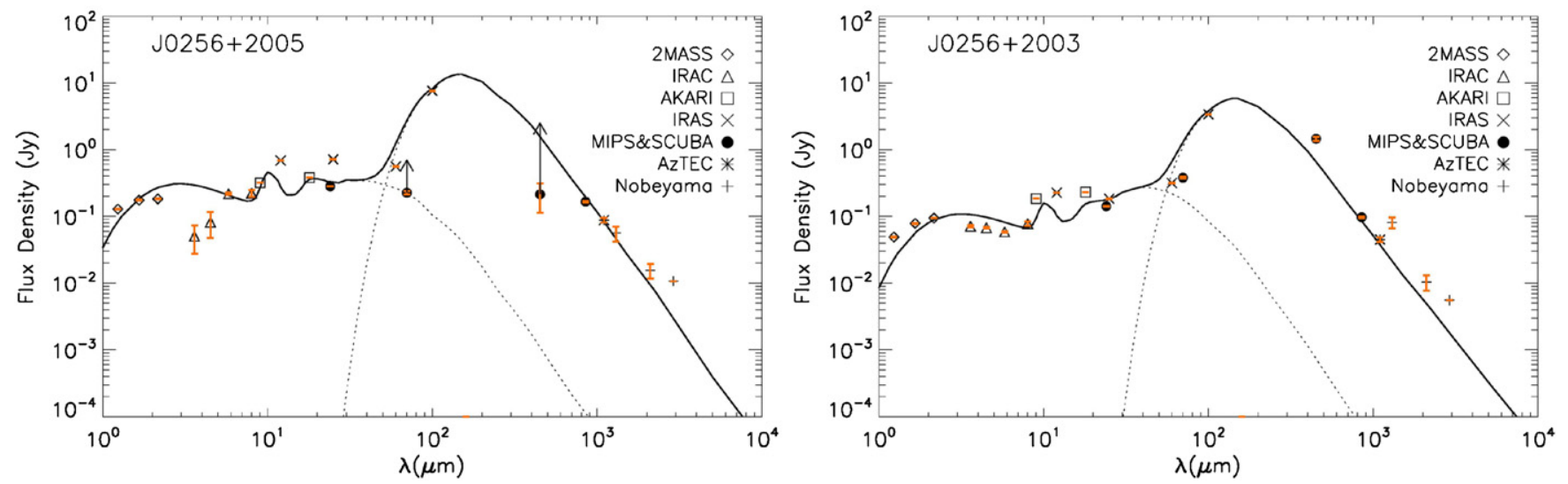

Figure 5. SEDs of two AzTEC sources corresponding to the candidates of protostars, J0256 + 2005 (left) and J0256 + 2003 (right).

(A color version of this figure is available in the online journal.)

Table 4

Fluxes at Other Wavelengths for the Detected AzTEC Sources

\begin{tabular}{|c|c|c|c|c|c|c|}
\hline Source & $\begin{array}{c}\text { 2MASS } J(1.25 \mu \mathrm{m}) \\
(\mathrm{mJy})\end{array}$ & $\begin{array}{c}\text { 2MASS } H(1.65 \mu \mathrm{m}) \\
(\mathrm{mJy})\end{array}$ & $\begin{array}{c}\text { 2MASS } K(2.17 \mu \mathrm{m}) \\
(\mathrm{mJy})\end{array}$ & $\begin{array}{l}S_{1.4 \mathrm{GHz}} \\
(\mathrm{mJy})\end{array}$ & $z$ & Ref. \\
\hline $\mathrm{J} 0255+1904$ & $(<) 6.05 \pm 0.097$ & $(<) 18.0 \pm 0.306$ & $(<) 21.3 \pm 0.341$ & & & \\
\hline $\mathrm{J} 0256+2005$ & $129.0 \pm 2.064$ & $175.0 \pm 2.975$ & $183.0 \pm 1.994$ & & & $1,2,6,8$ \\
\hline $\mathrm{J} 0256+2003$ & $49.0 \pm 0.784$ & $78.5 \pm 1.335$ & $94.6 \pm 1.031$ & & & $1,5,6,7$ \\
\hline $\mathrm{J} 0258+2030$ & $0.663 \pm 0.097$ & $1.04 \pm 0.067$ & $1.62 \pm 0.08$ & $98.1 \pm 3.0$ & & 2,3 \\
\hline J0259+1925 & $(<) 6.05 \pm 0.097$ & $(<) 18.0 \pm 0.306$ & $(<) 21.3 \pm 0.341$ & $163.7 \pm 4.9$ & 0.54 & 2,9 \\
\hline J0258+1953 & $(<) 6.05 \pm 0.097$ & $(<) 18.0 \pm 0.306$ & $(<) 21.3 \pm 0.341$ & $98.1 \pm 3.0$ & & \\
\hline $\mathrm{J} 0253+1805$ & $(<) 6.05 \pm 0.097$ & $(<) 18.0 \pm 0.306$ & $(<) 21.3 \pm 0.341$ & $304.0 \pm 9.1$ & 0.427 & $2,4,10$ \\
\hline $\mathrm{J} 0257+1847$ & $(<) 6.05 \pm 0.097$ & $(<) 18.0 \pm 0.306$ & $(<) 21.3 \pm 0.341$ & $103.7 \pm 3.1$ & & $2,3,4,11$ \\
\hline
\end{tabular}

References. (1) Cutri et al. 2003; (2) Condon et al. 1998; (3) Gregory et al. 1996; (4) Snellen et al. 2002; (5) Herbig \& Bell 1988; (6) Fernández et al. 1995; (7) Magnani et al. 1995; (8) Gameiro et al. 1993; (9) Caccianiga et al. 2000; (10) Ma et al. 1998; (11) Beasley et al. 2002.

1.4 GHz. The counterpart corresponding to J0259+1925 (A4 $\left.4_{1}\right)$ was also classified as J025929+1925.7 (1REX) in the radio emitting X-ray sources (REXs) survey by Caccianiga et al. (2000). Its X-ray flux was reported as $2.34 \times 10^{-13} \mathrm{erg} \mathrm{s}^{-1} \mathrm{~cm}^{-2}$. The NRAO VLA Sky Survey (NVSS) position of this source suggests that this object should be $\alpha=02^{\mathrm{h}} 59^{\mathrm{m}} 29^{\mathrm{s}} .65, \delta=$ $+19^{\circ} 25^{\prime} 44^{\prime \prime} .9$ (J2000) at radio wavelengths. From the present AzTEC observations, the position of J0259+1925 was measured as $\alpha=02^{\mathrm{h}} 59^{\mathrm{m}} 29^{\mathrm{s}} .5, \delta=+19^{\circ} 25^{\prime} 42^{\prime \prime}$ at millimeter wavelengths. So the difference between the radio and millimeter emission is $\Delta \alpha=0.15$ and $\Delta \delta=2.9$. The flux at $1.1 \mathrm{~mm}$ measured with AzTEC was $234.3 \mathrm{mJy}$ beam $^{-1}$. The AzTEC source A9 1 corresponds to J0253+1805 (J2000) in the NRAO VLA sky survey at $1.4 \mathrm{GHz}$ (Condon et al. 1998). The coordinate of $\mathrm{J} 0253+1805\left(\mathrm{~A} 9_{1}\right)$ was reported as $\alpha=02^{\mathrm{h}} 53^{\mathrm{m}} 34^{\mathrm{s}} .88$, $\delta=+18^{\circ} 05^{\prime} 42^{\prime \prime} .53$ (J2000) using the VLA observations. This position agrees with the identification by the Automated Plate Measurement (APM) Facility at Cambridge of the radio sources from the Jodrell Bank VLA Astrometric Survey (JVAS) (Snellen et al. 2002). We also compared the fluxes at $6 \mathrm{~mm}$ (Green Bank), NVSS 1.4 GHz (NRAO VLA Sky Survey), Green Bank $1.4 \mathrm{GHz}$, and VLA $1.4 \mathrm{GHz}$. The results were, respectively, 248, 304, 426, and $154 \mathrm{mJy}$. The millimeter-wavelength flux from this study was $298.5 \mathrm{mJy}^{\text {beam }^{-1}}$ at $1.1 \mathrm{~mm}$. The AzTEC source detected as $\mathrm{J} 0257+1847\left(\mathrm{~A} 9_{2}\right)$ has its counterpart in the radio source in the NRAO VLA sky survey by Condon et al.
(1998). This source was also included in the Very Long Baseline Array (VLBA) Calibrator Survey (VCS1) at 2.3 and $8.4 \mathrm{GHz}$ (Beasley et al. 2002). Snellen et al. (2002) also obtained the flux densities of this object at $6 \mathrm{~mm}$ (Green Bank), $1.4 \mathrm{GHz}$ (NVSS), 1.4 GHz (Green Bank), and 1.4 GHz (VLA) as 209, 103, 180, and $241 \mathrm{mJy}$, respectively. Synchrotron properties and the spectral indices of these objects will be discussed in detail in a future paper (S. Kim et al., in preparation). The coordinates of the AzTEC source agree to within $\alpha=$ $02^{\mathrm{h}} 57^{\mathrm{m}} 45^{\mathrm{s}} .6, \delta=+18^{\circ} 47^{\prime} 06^{\prime \prime}(\mathrm{J} 2000)$. The flux at $1.1 \mathrm{~mm}$ is $53.6 \mathrm{mJy}$ beam $^{-1}$. The fluxes at other wavelengths were compared in the SEDs presented in Figure 6. Although two AzTEC sources, J0225+1904 (A1 $\left.1_{1}\right)$ and J0258+1953 (A4 $\left.4_{2}\right)$, do not have counterparts at other wavelengths, we also performed a stacking analysis (Scott et al. 2008) on these AzTEC detections to calculate upper limits of the fluxes at the near-infrared images (Table 4). The GRASIL model (Silva et al. 1998) computes the photometric evolution of galaxies based on the SEDs of the AzTEC sources, including the effects of a dusty interstellar medium. The model includes the escape time of young stars from molecular clouds, the fraction of residual gas in molecular clouds, the radius of molecular clouds, etc. The star formation rate (SFR) could be also derived by fitting the SEDs to the model (Kim et al. 2010). The results suggest that J0258+2030 (A3 $)$, J0253+1805 (A91), and J0257+1847 (A92) can have SFRs of $3.6 \times 10^{3} M_{\odot} \mathrm{yr}^{-1}, 3.3 \times 10^{4} M_{\odot} \mathrm{yr}^{-1}$, and $2.9 \times 10^{2} M_{\odot} \mathrm{yr}^{-1}$, 

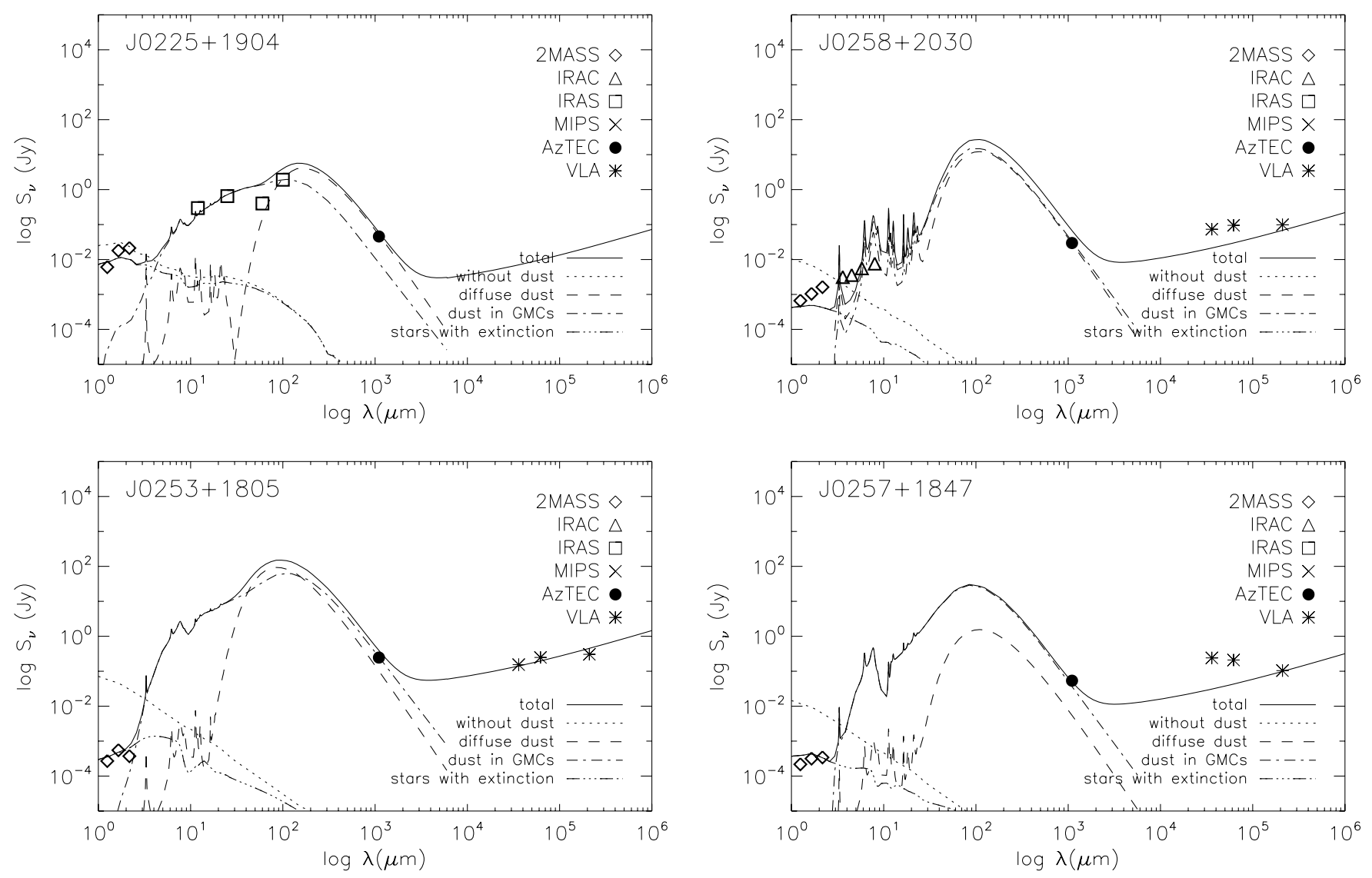

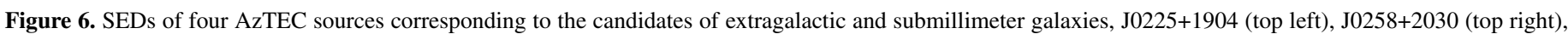
J0253+1805 (bottom left), and J0257+1847 (bottom right).

respectively. The SFRs are similar to those studied in 16 radio galaxies with AzTEC counterparts in the far-IR and a graybody emission template with an emissivity index, $\beta$, and a dust temperature, $T_{d}$ (Humphrey et al. 2011).

\section{CONCLUSION}

We presented observations of the dust continuum emission at $1.1 \mathrm{~mm}$ from MBM12 performed with AzTEC at the JCMT. We surveyed about $6.34 \mathrm{deg}^{2}$ field of sky. This is the largest area that has ever been surveyed for the MBM12 region with a (sub-)millimeter telescope. The distribution of $1.1 \mathrm{~mm} \mathrm{AzTEC}$ sources is spatially anti-correlated with that of the $100 \mu \mathrm{m}$ emission and that of the ${ }^{12} \mathrm{CO}$ emission. We detected 312 sources with a $\mathrm{S} / \mathrm{N}$ of over $3 \sigma$. The FDR was $319 \pm 66$. Eight sources were detected with a $\mathrm{S} / \mathrm{N}>4.4 \sigma$ and with FDR value smaller than 1. These eight AzTEC sources can be considered to be real astronomical objects as opposed to spurious detections. We detected $1.1 \mathrm{~mm}$ sources associated with two classical T Tauri stars, $\mathrm{LkH} \alpha 262$ and $\mathrm{LkH} \alpha 264$. Observations of their SEDs indicate that $\mathrm{LkH} \alpha 262$ is likely to be Class II but may be a late Class I. A flared disk and a bipolar cavity in models of Class I sources lead to more complicated SEDs. Our detections suggest that at least the classical T Tauri stars in MBM12 have disks in a similar mass range to the disks of stars in the Taurus or $\rho$ Ophiuchus regions. From the present AzTEC observations of the MBM12 region, we find that some of the other detected sources are likely to be located behind the MBM12 molecular cloud. These have radio counterparts, and their SFRs were derived from fits of their SEDs to the photometric evolution of galaxies when the effects of a dusty interstellar medium were included. These SFRs ranged from a few hundred to a few thousand $M_{\odot} \mathrm{yr}^{-1}$. Direct measurements of their redshifts should be performed with the Large Millimeter Telescope in the near future.

We thank K. Scott, J. Lowenthal, and other AzTEC team members for their contributions to the project. We also thank S. Johnson for helpful suggestions on the manuscript. This research has made use of the NASA/IPAC Extragalactic Database (NED) which is operated by the Jet Propulsion Laboratory, California Institute of Technology, under contract with the National Aeronautics and Space Administration. This publication has made use of data products from the Two Micron All Sky Survey, which is a joint project of the University of Massachusetts and the Infrared Processing and Analysis Center/California Institute of Technology, funded by the National Aeronautics and Space Administration and the National Science Foundation. This research has made use of the NASA/IPAC Infrared Science Archives, which is operated by the Jet Propulsion Laboratory, California Institute of Technology, under contract with the National Aeronautics and Space Administration. M.K. was supported in part by Basic Science Research Program through the National Research Foundation of Korea (NRF) funded by the Ministry of Education, Science and Technology 2009-0066892. This research was supported in part by Mid-career Researcher Program through the National Research Foundation of Korea (NRF) funded by the Ministry of Education, Science and Technology 2011-0028001. We thank the anonymous referee for very helpful comments on the manuscript. 


\section{REFERENCES}

Andersson, B.-G., Idzi, R., Uomoto, A., et al. 2002, AJ, 124, 2164

André, P., Ward-Thompson, D., \& Barsony, M. 1993, ApJ, 406, 122

Andrews, S. M., \& Williams, J. P. 2007, ApJ, 671, 1800

Austermann, J. E., Dunlop, J. S., Perera, T. A., et al. 2010, MNRAS, 401, 160

Beasley, A. J., Gordon, D., Peck, A. B., et al. 2002, ApJ, 141, 13

Broeg, C., Joergens, V., Fernández, M., et al. 2006, A\&A, 450, 1135

Brunt, C. M., \& Heyer, M. H. 2002, ApJ, 566, 276

Caccianiga, A., Maccacaro, T., Wolter, A., Della Ceca, R., \& Gioia, I. M. 2000, A\&A, 114, 247

Caillault, J.-P., Magnani, L., \& Fryer, C. 1995, ApJ, 441, 261

Condon, J. J., Cotton, W. D., Greisen, E. W., et al. 1998, AJ, 115, 1693

Cutri, R. M., Skrutskie, M. F., van Dyk, S., et al. 2003, The IRSA 2MASS All-Sky Point Source Catalog, NASA/IPAC Infrared Science Archive

Enoch, M. L., Glenn, J., Evans, N. J., II, et al. 2007, ApJ, 666, 982

Feigelson, E. D. 1996, ApJ, 468, 306

Fernández, M., Ortiz, E., Eiroa, C., \& Miranda, L. F. 1995, A\&AS, 114, 439

Fleming, T. A., Gioia, I. M., \& Maccacaro, T. 1989, ApJ, 340, 1011

Francis, P. J., \& Wills, B. J. 1999, in ASP Conf. Ser. 162, Quasars and Cosmology, ed. G. Ferland \& J. Baldwin (San Francisco, CA: ASP), 363

Gameiro, J. F., Lago, M. T. V. T., Lima, N. M., \& Cameron, A. C. 1993, MNRAS, 261, 11

Gregory, P. C., Scott, W. K., Douglas, K., \& Condon, J. J. 1996, ApJS, 103, 427

Hearty, T., Fernández, M., Alcalá, J. M., et al. 2000b, A\&A, 357, 681

Hearty, T., Neuhäuser, R., Stelzer, B., et al. 2000a, A\&A, 353, 1044

Herbig, G. H., \& Bell, K. R. 1988, Lick Observatory Bulletin, IIII, 1

Herbst, W., Williams, E. C., \& Hawley, W. P. 2004, AJ, 127, 1594

Heyer, M. H., \& Schloerb, F. P. 1997, ApJ, 475, 173
Hogerheijde, M. R., Jayawardhana, R., Johnstone, D., Blake, G. A., \& Kessler, J. E. 2002, AJ, 124, 3387

Hogerheijde, M. R., Johnstone, D., Matsuyama, I., Jayawardhana, R., \& Muzerolle, J. 2003, ApJ, 593, L101

Humphrey, A., Zeballos, M., Aretxaga, I., et al. 2011, MNRAS, 418, 74

Itoh, Y., Sugitani, K., Fukuda, N., et al. 2003, ApJ, 586, 141

Ivezic, Z., Nenkova, M., \& Elitzur, M. 1999, User Manual for DUSTY, University of Kentucky Internal Report (arXiv:astro-ph/9910475)

Kim, S., Kwon, E., Madden, S. C., et al. 2010, A\&A, 518, 75

Laurent, G. T., Aguirre, J. E., Glenn, J., et al. 2005, ApJ, 623, 742

Luhman, K. L. 1999, ApJ, 525, 466

Luhman, K. L. 2001, ApJ, 560, 287

Lynds, B. T. 1962, ApJS, 7, 1

Ma, C., Arias, E. F., Eubanks, T. M., et al. 1998, AJ, 116, 516

Magnani, L., Blitz, L., \& Mundy, L. 1985, ApJ, 295, 402

Magnani, L., Caillault, J.-P., Buchalter, A., \& Beichman, C. A. 1995, ApJS, 96, 159

Meeus, G., Juhász, A., Henning, Th., et al. 2009, A\&A, 497, 379

Ossenkopf, V., \& Henning, Th. 1994, A\&A, 291, 943

Perera, T. A., Chapin, E. L., Austermann, J. E., et al. 2008, MNRAS, 391, 1227

Pound, M. W., Wilson, R. W., \& Bania, T. M. 1990, ApJ, 351, 165

Scott, K. S., Austermann, J. E., Perera, T. A., et al. 2008, MNRAS, 385, 2225

Silva, L., Granato, G. L., Bressan, A., \& Danese, L. 1998, ApJ, 509, 103

Snellen, A. G., McMahon, R. G., Hook, I. M., \& Browne, I. W. 2002, MNRAS, 329,700

Sterzik, M. F., \& Durisen, R. H. 1995, A\&A, 304, L9

Whitney, B. A., Wood, K., Bjorkman, J. E., \& Cohen, M. 2003, ApJ, 598, 1079

Williams, J. P., \& Cieza, L. A. 2011, ARA\&A, 49, 67

Wilson, G. W., Austermann, J. E., Perera, T. A., et al. 2008, MNRAS, 386, 807 